\title{
28 \\ A Long Term Initiative in the Development of I.T. Tools for Decision Support
}

\author{
C. M. Khoong \\ Information Technology Institute \\ 11 Science Park Road, Singapore 117685, Republic of Singapore \\ Email: chanmeng@iti.gov.sg
}

\begin{abstract}
IT tools for decision support are often the key differentiating factors in highly integrated management systems today. However, the deployment of such tools has been hampered by the high costs of skilled analysts and the technology diffusion gaps between research institutions and the IT industry. We present a comprehensive architecture that is used as a basis for a long term R\&D programme on decisions support tools. We also describe the development and diffusion strategies applied to these tools.
\end{abstract}

\section{Keywords}

Decision support systems, enterprise modelling, reengineering, research and development, technology diffusion.

\section{INTRODUCTION}

Decision support is the capability to assist managerial and operational decision makers in transforming information into useful courses of action for the organization. IT tools for decision support, often called decision support systems, utilize methods of information modeling, scenario analysis, decision optimization, and human-computer interfaces. Increasingly, decision support tools are being embedded in large, networked information systems providing total integration of the organization's business processes. While the decision support component may physically be a small and sometimes invisible function of such systems, it is also usually the key differentiating factor and generator of competitive advantage for users.

Unfortunately, there appears to be a high cost associated with the development and deployment of advanced decision support tools. The high cost of manpower in the IT industry 
in general is a cause for this. Another cause is the technology diffusion gap between research institutions and the IT industry. The latest and the best techniques for decision support come from academia. However, research environments lack the infrastructure and motivation for transforming the science into robust technology components in software. On the other hand, the IT industry is hungry for the innovations, but lack the luxury of time or money to invest in research.

The author's institute is in the unique position (and difficult role) of bridging the technology diffusion gap. Being an applied IT research institute, we seek to find industrial applications for advanced technologies. Decision support technology is an area of focus for the institute. A decision support technology group has been established and tasked with bridging the diffusion gap between researchers and practitioners. More specifically, the deliverables from this group should prepare applications developers technically for their future roles in large-scale, industry-wide and national projects. This paper describes the envisaged technical architecture for decision support tools, the development and deployment strategy, and the progress of the implementation project.

\section{TECHNICAL ARCHITECTURE}

The technical architecture describes the spectrum of decision support components to be realized in software, linkages among the components, and tools for integrating the components into systems. A multi-layered architecture has been envisaged, in which each layer provides the components and/or mechanisms for integration into the next layer. The layers are: Decision Support Components Library, DSS Development Workbench, Systems, Enterprise Modeling/ BPR, and Application Sectors. The technical architecture is depicted in Figure 1. We explain each layer below.

The Decision Support Components Library is a collection of coded techniques for decision support functions. The library includes codes and pseudocode templates for: data structures, linear programming, network optimization, combinatorial search, cutting/packing/ partitioning, forecasting/ data analysis, knowledge bases, reasoning, and statistics. The purpose of the library is to facilitate rapid construction of decision support systems. Some of the components in the library are commercial tools (e.g. linear programming), while others are adapted from public domain software or generalized from previous system development projects. The library is continually being extended.

The DSS Development Workbench is intended to provide a semi-automated environment for the assembly of decision support systems to meet specified requirements for decision support. Some concepts of DSS workbenches are discussed in (Raghavan, 1992). We do not believe that a fully automated workbench is technically feasible, as every decision support system (unlike office productivity tools like word processors and spreadsheets) has its own peculiar objectives, constraints, and assumptions. The automated help that the workbench can provide is in matching decision problem statements with candidate solution techniques found in the Components Library. The matching process is guided by the knowledge of a DSS taxonomy.

The Systems layer comprises the suite of decision support systems that have been developed. The systems may be in the form of generic, customizable tools, demonstrable prototypes, or applications deployed in industry. The systems are therefore created with both 
technology-driven and market-driven motivations. We believe that such a portfolio is pragmatic for our initiative. The continual check-and-balance between the two perspectives gives the best chance of achieving the technology diffusion goal.

The Enterprise Modeling/ BPR layer provides the mechanisms for integration of decision support systems into the business processes of the organization. An enterprise model encodes the interplays among the organizational structure, its data and resources, and its processes and policies, and provides automated support for organizational coordination, intelligence, continuous improvement, and reengineering. The model stipulates where and how decision support systems are deployed in the organization, and the process by which the systems are executed and maintained.

The Applications Sectors layer describes requirements for decision support technologies in various industrial sectors. In the context of Singapore, the sectors with envisaged needs for advanced decision support over the next several years include land transport, business logistics, construction and real estate, government/ defense, and manufacturing. Structured specification of domain knowledge and decision support requirements in these sectors are the basis for the roll-out of future industry-wide and national IT projects. The specification will also describe how enterprise models may be linked and synergized.

\section{DEVELOPMENT AND DIFFUSION STRATEGIES}

The technical architecture could have been developed in a top-down manner. In this case, the architectural blueprint is defined in advance of any decision support application, and proposes components that are expected to find future applications. Another approach is bottom-up, in which the technical architecture is empty at the beginning but gets filled opportunistically from various components development endeavors. The top-down approach suffers from weak grounding in the real world, while the bottom-up approach suffers from lack of focus and coordination in technical efforts.

We have therefore adopted the compromise middle-out approach to the realization of the technical architecture. Our starting point was our many years of experience in developing decision support systems for industry. Based on this experience, and field studies of current and future requirements in industry for decision support technologies, the first version of the technical architecture was released. This first version was used to focus the efforts in identifying new industrial applications and research projects. The results of the identification exercises and (in follow-through cases) of research and applications, served as feedback on the overall architecture. The architecture described in this paper is in the second version. The first version of the architecture was defined in terms of system frameworks for manpower/service management and logistics management (Khoong, 1995c).

Over the next few years, our technology diffusion strategy for the decision support tools will take the form depicted in Figure 2. The outputs of fundamental research in decision science and related disciplines will flow into the institute through joint research collaborations with universities. We then turn the science into concrete concepts for software components. These 'proto-components' undergo rounds of validation on testbeds from realworld environments before stabilizing as robust and reusable components. We refer to the generation of technology components from science as a technology development (TD) process. This process constitutes a core competence of our institute. 
The diffusion of the technology components can happen through several channels. The components may be integrated into applications developed by the institute. These applications are likely to be part of national projects, for which indigenous development capability is essential. The components can also be integrated into applications developed by industrial partners. These are likely to be organizations with mature, in-house software development capability. Yet another diffusion channel is through product developers and vendors, who can license the components for integration into their commercial offerings. While diffusion through applications development (AD) is targeted at a few specific organizations, diffusion through product development (PD) depends on a wide market base.

Increasingly though, we see our competence in decision support tools being manifested as services instead of products or components. The consultancy services will offer analysis and design expertise, possibly using our tools to provide back-end support for delivering the expertise. In the long run, we see some of our services being delivered electronically, via the World Wide Web. Web-based services create entirely new marketplaces for decision support tools. Some results in this direction have been reported by some research laboratories (Bhargava, 1995). Electronic services are attractive because consumers need only to pay for solutions as and when needed, without investing on expensive DSS development projects. Users only need high speed network links and good web browsers to tap on the services, rather than high end computing workstations. For service providers like our institute, the electronic medium expands the diffusion bandwidth tremendously with little additional cost.

\section{CURRENT DEVELOPMENTS}

The diffusion strategies translate are translated into a portfolio of R\&D projects that are currently being undertaken. In particular, as a preparation for sector integration, the Systems layer in the technical architecture is currently focused on the following classes: Dynamic Layout Systems, Routing and Scheduling Systems, Distribution Planning Systems, and Competitive Gaming Systems. Figure 1 shows a sampling of tools that may be developed within each class.

Dynamic Layout Systems (DLS) assist decision makers with designing and managing arrangements of facilities, resources, products, and services over time and space. Applications of DLS include berth allocation in seaports, gate assignment at airports, facility layout in factories, warehouse space management, and VLSI chip design. Much of our practical experience in DLS is being derived from the development of a large-scale DLS for the seaport authority in Singapore. This particular project incorporates several alternative combinatorial optimization techniques coded in the $\mathrm{C}++$ language, and integrated with organizational data capture and operations control systems. In this project, many decision subproblems were uncovered, each of which has spun off separate research projects that would eventually yield generic DLS components (Heng et al, 1996a).

Routing and Scheduling Systems (RSS) assist decision makers with the routing and scheduling of resources to serve activities over time and space. We have developed many RSS for organizations in the business logistics industry, including manpower rostering (Khoong et al, 1994b), crew deployment, vehicle scheduling, course timetabling, production planning, shipment delivery planning, and maintenance scheduling tools. We are seeking to 
generalize these tools as much as possible, and to transfer some core algorithms to the Components Library.

Distribution Planning Systems (DPS) assist decision makers in planning the distribution of resources over time and space to meet future needs of these resources. We have developed industrial DPS for domains such as container movements in the shipping industry (Shen \& Khoong, 1995), long term manpower planning for large organizations (Khoong, 1996a), and ammunition management for the defense industry. We are generalizing these models for the instantiation of other DPS, such as global supply chain management systems.

Competitive Gaming Systems (CGS) assist decision makers in planning courses of action for organizational resources in multi-player, dynamic, uncertain, and possibly hostile environments. Group and distributed decision support systems may be considered as subclasses of CGS. We have not developed any CGS at the moment, but some conceptual foundations have been laid for CGS applications in concrete domains. We believe that CGS represent an important future wave of decision support technologies (Khoong, 1995a).

The Decision Support Components Library will be extended largely through endeavors in the Systems layer. We also engage regular streams of students and research assistants from local universities to help us in building the components. As for the DSS Development Workbench, we have devised a detailed taxonomy (Khoong, 1995b) for guiding the construction of systems. The next step would be to encode some of the guidance logic in software. In the Enterprise Modeling/ BPR layer, we have developed structured methodologies for reengineering (Khoong, 1996b) and DSS feasibility analysis (Khoong \& $\mathrm{Ku}, 1994 \mathrm{a})$. A research project has been initiated for the development of enterprise models for service organizations (Heng et al, 1996b).

A primary end target of the technical architecture is in industry-wide and national projects, for sectors identified in the Applications Sectors layer. Some sectorial initiatives are underway or being planned under the IT2000 national masterplan in Singapore. IT2000 projects span many years, and present many opportunities for the development of advanced decision support technologies. Our R\&D initiative serves the role of providing the technology lookahead for these mega-projects, and would equip applications developers in time with the technology components for the eventual systems.

In the land transport sector, we are working with national authorities, system integrators, and local universities on research projects in traffic management systems and travel advisory systems. In the business logistics sector we are working with the seaport authority and major freight forwarders on fleet scheduling and tracking systems. In the construction and real estate sector (codenamed 'CORENET' in Figure 1), we are working with regulatory agencies on systems for automated interpretation and checking of building plans, while systems for construction project management are in the pipeline. Opportunities to deploy decision support tools in other sectors, such as defence, manufacturing, and law and justice, are being monitored.

\section{CONCLUDING REMARKS}

We have presented a comprehensive technical architecture as the basis for long term $\mathrm{R} \& \mathrm{D}$ programme in decision support tools. We also discussed our development and diffusion strategies, and the achievements to date. We expect the architecture to continue to evolve as 
insights are derived from the marriage of research results with applications experience. We also hope that our initiative will contribute toward a cumulative tradition for decision support systems research (Eom, 1995).

\section{REFERENCES}

Bhargava, H. K., King, A. S. and McQuay, D. S. (1995) DecisionNet: an architecture for modeling and decision support over the World Wide Web. Proceedings of the ISDSS 3rd International Conference, Hong Kong, June 22-23, 499-505.

Eom, S. B. (1995) Decision support systems research: reference disciplines and a cumulative tradition. OMEGA International Journal of Management Science, 23, 511-523.

Heng, K. J., Khoong, C. M. and Lim, A. (1996a) A forward algorithmic strategy for largescale resource allocation systems. Proceedings of the 1st Asia-Pacific DSI Conference, Hong Kong, June 21-22, to appear.

Heng, K. J., Khoong, C. M. and Radjou, N. (1996b) An extended research programme on enterprise modeling. Submitted for publication.

Khoong, C. M. (1995a) Decision support systems: an extended research agenda. OMEGA International Journal of Management Science, 23, 221-229.

Khoong, C. M. (1995b) Toward DSS science: a taxonomic structure for R\&D. Proceedings of the ISDSS 3rd International Conference, Hong Kong, June 22-23, 419-431.

Khoong, C. M. (1995c) Intelligent system frameworks for service operations management, in OR Applications in Singapore (eds. M. Goh and L. C. Tang), Operations Research Society of Singapore, Singapore, 15-24.

Khoong, C. M. (1996a) An integrated system framework and analysis methodology for manpower planning. International Journal of Manpower, 17, $26-46$.

Khoong, C. M. (1996b) 'Second wave' reengineering: structure and methodology. Proceedings of the 1st Asia-Pacific DSI Conference, Hong Kong, June 21-22, to appear.

Khoong, C. M. and Ku, Y. W. (1994a) A holistic feasibility study framework for decision systems. IEEE Transactions on Systems, Man \& Cybernetics, 24, 100-106.

Khoong, C. M., Lau, H. C. and Chew, L. W. (1994b) Automated manpower rostering: techniques and experience. International Transactions in Operational Research, 1, 353361.

Raghavan, S. A. (1992) A high level framework for developing DSS workbenches. Proceedings of the 25th Hawaii International Conference on System Sciences, January 7-10, 505-516.

Sage, A. P. (1991) Decision Support Systems Engineering. John Wiley, New York.

Shen, W. S. and Khoong, C. M. (1995) A DSS for empty container distribution planning. Decision Support Systems, 15, 75-82.

Turban, E. (1993) Decision Support and Expert Systems: Management Support Systems, 3rd Edition. Macmillan, New York. 


\section{BIOGRAPHY}

Chan Meng Khoong is Programme Manager at the Information Technology Institute in Singapore, where he heads the development of advanced decision support tools. He is concurrently Manager of the Centre for Strategic Process Innovation at Singapore's National Computer Board. He is also appointed as Adjunct Senior Researcher at the National University of Singapore, to the Board of Directors at the Chartered Institute of Transport, to the Editorial Advisory Board of the Journal of Applied Management Studies, and as Area Editor with the International Journal of Operations and Quantitative Management. He has authored 80 publications in international journals and conferences.

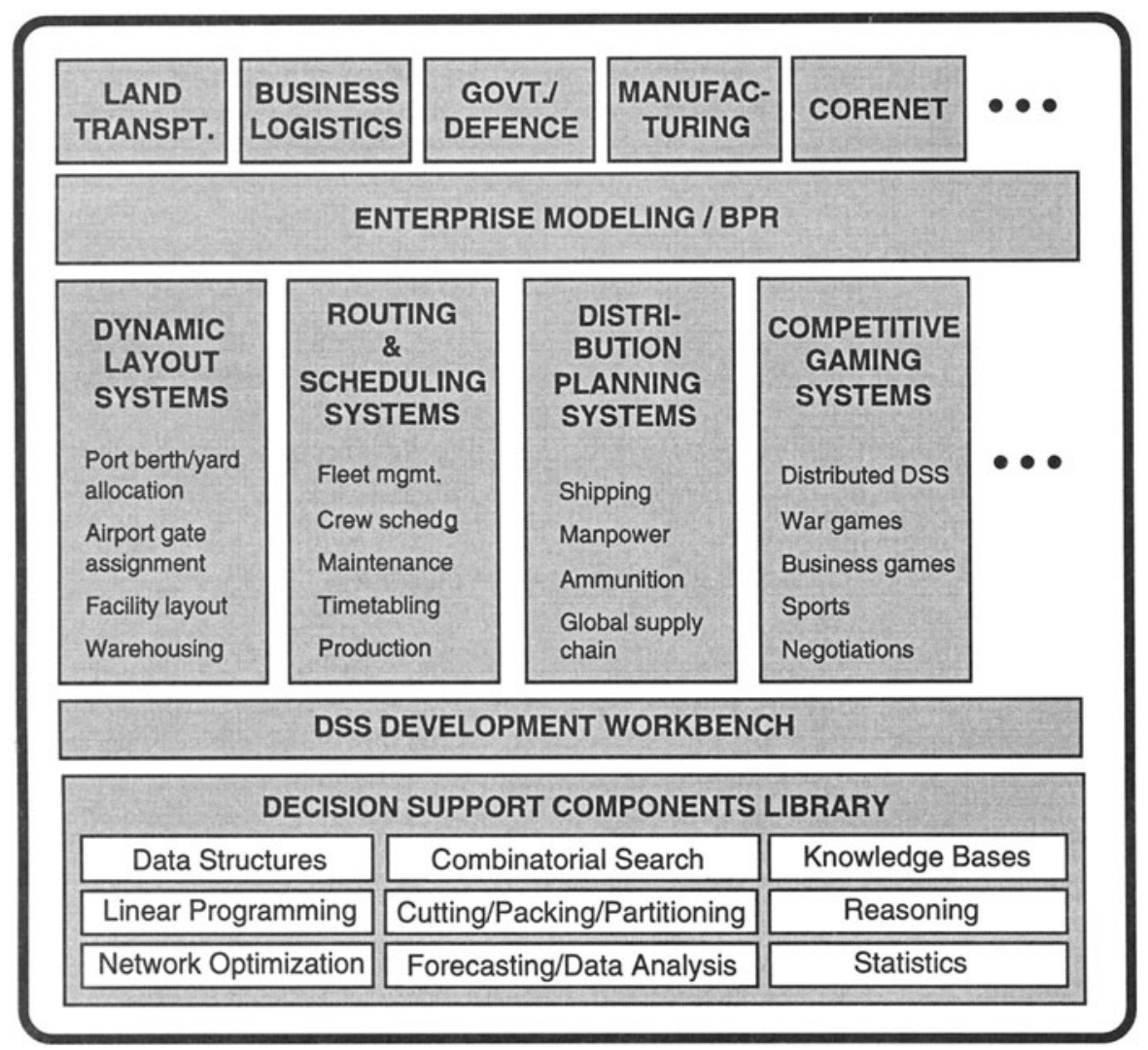

Figure 1 Technical architecture for decision support technologies. CORENET is a codename for the construction and real estate sector. 


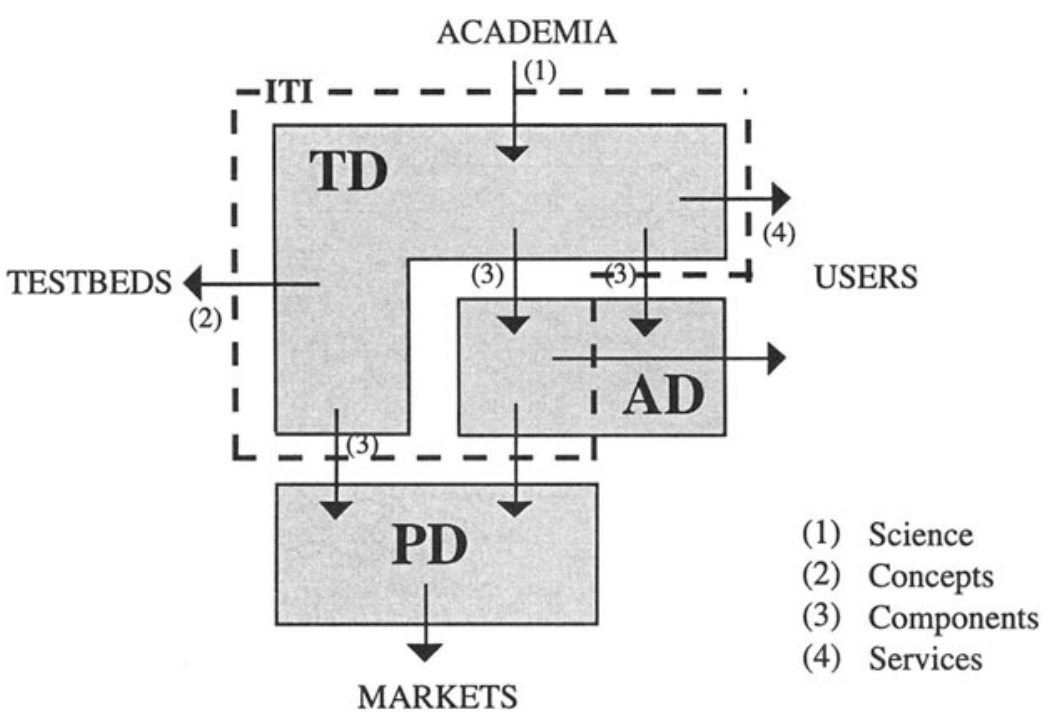

Figure 2 Technology diffusion strategy for decision support tools. The main activities undertaken in the Information Technology Institute (ITI) are technology development (TD) and some applications development (AD), while product development (PD) is normally undertaken by partners in the IT industry. 